FERMILAB-Conf-92/102

\section{Theoretical Methods for Creep and Stress Relaxation Studies of SSC Coil}

FNAL/C $-92 / 102$

DE93 000621
J. McAdams and F. Markley

Fermi National Accelerator Laboratory

P.O. Box 500, Batavia, Illinois 60510

April 1992

Presented at the Fourth Annual IISSC Conference, New Orleans, LA, March 4-6, 1992. 


\title{
THEORETICAL METHODS FOR CREEP AND STRESS RELAXATION STUDIES OF SSC COIL
}

\author{
James McAdams and Finley Markley \\ Fermilab National Accelerator Laboratory* \\ Box 500 \\ Batavia, IL 60510
}

\begin{abstract}
Extrapolation of laboratory measurements of SSC coil properties to the actual construction of SSC magnets requires mathematical models of the experimental data. A variety of models were used to approximate the data collected from creep and stress relaxation experiments performed on Kapton film and SSC coil samples. The coefficients for these mathematical models were found by performing a least-squares fit via the program MINUIT.

Once the semiempirical expressions for the creep data were found, they were converted to expressions for stress relaxation using an approximation of the Laplace integral relating the two processes. The data sets from creep experiments were also converted directly to stress relaxation data by numeric integration. Both of these methods allow comparison of data from two different methods of measuring viscoelastic properties.
\end{abstract}

Three companion papers presented at this conference will present:

1) Stress relaxation in SSC $50 \mathrm{~mm}$ dipole coil.

2) Measurement of the elastic modulus of Kapton perpendicular to the plane of the film at room and cryogenic temperatures.

3) Temperature dependence of the viscoelastic properties of SSC coil insulation (Kapton).

* Operated by the Universities Research Association, Inc., for the U.S. Department of Energy. 


\section{INTRODUCTION}

To prevent mechanical motion within the magnets during SSC operation, these magnets are manufactured so that the coils are under a compressive stress. However, any material which is constrained in such a manner will show a reduction in stress over time. Due to the long storage periods and operating lifetimes of the SSC magnets, this stress relaxation could be considerable, and must be accounted for to avoid a reduction of stress which would allow movement of the coil during cool-down and operation.

In order to predict the long-term effects of this stress relaxation, a variety of experiments were performed, as discussed in the companion papers to this one. The emphasis of this paper will be the detailing of the theoretical analysis used in the interpretation of data from these experiments. The most important of these procedures is the calculation of the Time-Temperature Superposition factor (allowing viscoelastic data to be extrapolated to different temperatures or intervals), the conversion of data among viscoelastic functions, and finding accurate models to describe the behavior of viscoelastic functions.

\section{METHODS}

In order to extrapolate data from relatively short term viscoelastic experiments to long term effects, some method of deriving an equation describing viscoelastic behavior from the creep data must be used. To test various models and methods of fitting them to data sets, we selected a representative data set from the work of Lautenschlager ${ }^{5}$ and compiled a list of possible forms for the viscoelastic function from the available literature.

To fit the models to the data set, we tried three methods: $\log$-log plots, selecting an appropriate number of likely data points and solving the model as $\mathrm{N}$ equations in $\mathrm{N}$ unknowns, and by the least-squares method using CERN's function minimization program MINUIT. We found that the best fits to the data set were consistently given by the MINUIT program, and performed all further work using this method of fitting models to data exclusively.

Once we had a standard method, we used this method to examine a variety of potential models. The models which we tried were:

$$
\begin{aligned}
& \varepsilon=\varepsilon_{0}+\varepsilon_{1} \mathrm{t}^{\mathrm{n}} \\
& \varepsilon=\varepsilon_{0}+\varepsilon_{\varepsilon} \mathrm{t}^{\mathrm{n}}+\varepsilon_{2} \mathrm{t} \\
& \varepsilon=\varepsilon_{0}+\varepsilon_{1} \ln (\mathrm{t}) \\
& \varepsilon=\varepsilon_{0}-\varepsilon_{0} \mathrm{e}^{\left(-t / \varepsilon_{1}\right)}, \text { the Voight model of viscoelasticity. } \\
& \varepsilon=\varepsilon_{0}+\varepsilon_{1} \mathrm{t}+\varepsilon_{2} \mathrm{t}^{2}
\end{aligned}
$$


where $\varepsilon_{0}, \varepsilon_{1}, \varepsilon_{2}, \varepsilon_{3}$, and $\mathrm{n}$ are coefficients which are to be varied to provide the best fit to observed data. All of these equations, except the polynomial equation, have been reported to have been used successfully to model creep data1,2,4. The results of the fittings are displayed in Figure 1. For the arbitrary data set selected, the best model of creep behavior is $\varepsilon=\varepsilon_{0}+\varepsilon_{1} \mathrm{t}^{\mathrm{n}}$.

One complication of this work on finding useful models for viscoelastic behavior is that a large set of values for the unknowns of our chosen model equation exist which give similar results as shown in Figure 2. Therefore the results of this parameterization should only be taken as approximate solutions, and may vary considerably. More confidence can be placed in the parameterization if any one of the terms (such as $\varepsilon_{0}$, the timeindependent strain) can be accurately determined, since this limits the range over which the other terms can be varied.

The second goal of our work was to find a method which would allow us to obtain a model of stress relaxation behavior based on data gathered from creep experiments.

\section{Comparison of Creep Models}

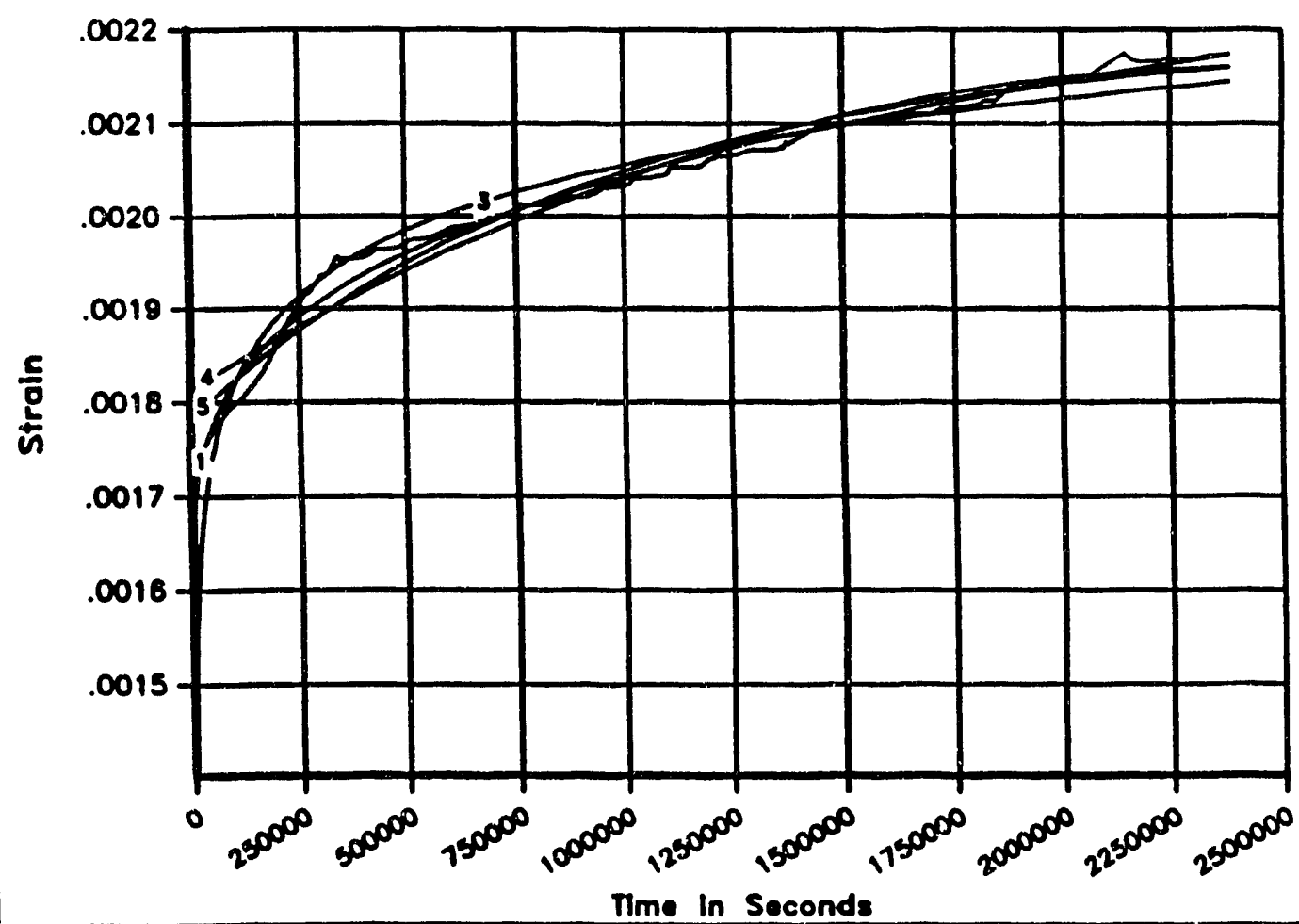

Figure 1: Comparison of Creep Models. 


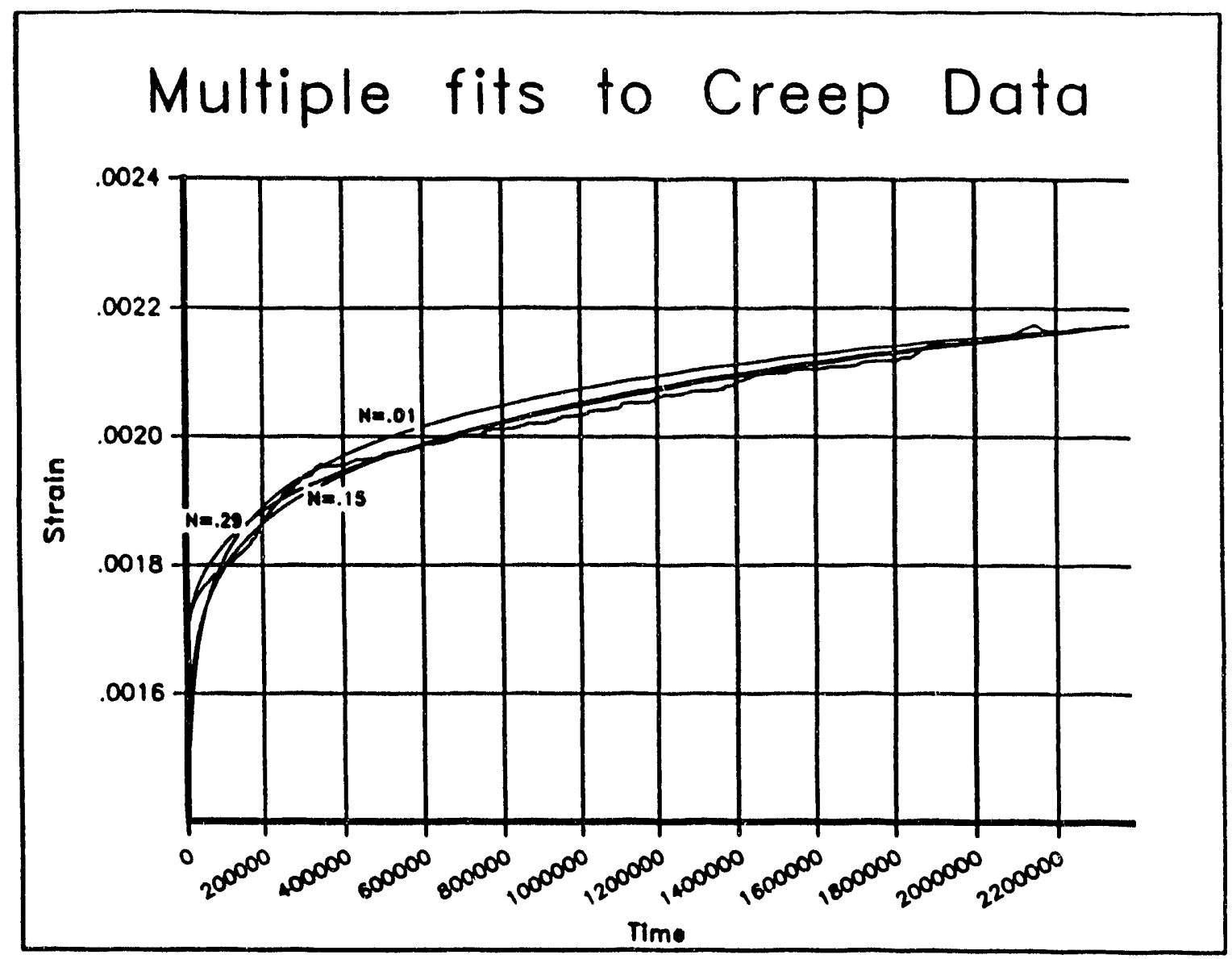

Figure 2: Varying parameters of the model.

The relationship between creep and stress relaxation is given by the convolution integral $\int(E(\tau) \mathrm{D}(\mathrm{t}-\tau) \mathrm{dT}=t$, or alternately by the LaPlacian expression $L[D] L[E]=1 / p^{2}$. Attempting to solve the LaPlacian expression requires that a function for either $D$ or $E$ is known.

Having chosen the expression $\varepsilon=\varepsilon_{0}+\varepsilon_{1} \mathrm{t}^{\mathrm{n}}$ as the best representation of creep behavior, we now proceed with solving the LaPlacian relationship for an expression of stress relaxation behavior.

We start with the form of our creep model, the LaPlacian relationship between the tensile modulus and tensile compliance, and the definition of compliance:

$$
L[E] L[D]=\frac{1}{p^{2}} \quad \varepsilon=\varepsilon_{0}+\varepsilon_{1} t^{n} \quad \varepsilon=\sigma D
$$

assuming that $\sigma$ is a constant, $D=D_{0}+D_{1} t^{n}$, where $D_{0}=\frac{\varepsilon_{0}}{\sigma}$ and $D_{1}=\frac{\varepsilon_{1}}{\sigma}$.

substituting, we find: $L\left(D_{0}+D_{1} t^{n}\right]=\frac{1}{L(E) p^{2}}$ 


$$
\begin{gathered}
L\left(\mathrm{D}_{0}\right\}+\mathrm{L}\left\{\mathrm{D}_{1} \mathrm{t}^{\mathrm{n}}\right\}=\frac{1}{\mathrm{~L}\{\mathrm{E}\} \mathrm{p}^{2}} \\
\frac{\mathrm{D}_{0}}{\mathrm{p}}+\mathrm{D}_{1} \frac{\Gamma(\mathrm{n}+1)}{\mathrm{p}^{\mathrm{n}+1}}=\frac{1}{\mathrm{~L}\{\mathrm{E}\} \mathrm{p}^{2}} \\
L\{E\}=\frac{1}{p^{2}}\left(\frac{D_{0}}{p}+D_{1} \frac{\Gamma(n-1)}{p^{n+1}}\right) \\
L\{E\}=\frac{1}{\left(D_{O} P+D_{1} P^{2} \frac{\Gamma(n+1)}{P^{n+1}}\right)} \\
L\{E\}=\frac{1}{D_{0} \mathrm{p}} \frac{1}{1+\frac{D_{1}}{D_{0}}(\mathrm{p}) \frac{\Gamma(\mathrm{n}+1)}{\mathrm{p}^{\mathrm{n}+1}}}
\end{gathered}
$$

At this point we found no way of proceeding with the evaluation of the anti-LaPlacian of the term on the right, and introduced the following approximation in order to proceed:

$$
1 /(1+\delta) \sim 1-\delta \quad \text { for } \delta \ll 1
$$

Using this, we convert the expression for $L(E)$ to:

$$
\begin{gathered}
\mathrm{L}\{\mathrm{E}\}=1 / \mathrm{D}_{\mathrm{o}} \mathrm{p}\left(1-\frac{\mathrm{D}_{1}}{\mathrm{D}_{0}}(\mathrm{p}) \frac{\Gamma(\mathrm{n}+1)}{\mathrm{p}^{\mathrm{n}+1}}\right) \\
\mathrm{L}\{\mathrm{E}\}=1 / \mathrm{D}_{\mathrm{o}} \mathrm{p}-\frac{\mathrm{D}_{1}}{\mathrm{D}_{0}^{2}} \frac{\Gamma(\mathrm{n}+1)}{\mathrm{p}^{\mathrm{n}+1}} \\
\mathrm{E}=1 / \mathrm{D}_{\mathrm{o}}-\frac{\mathrm{D}_{1}}{\mathrm{D}_{0}{ }^{2}} \mathrm{t}^{\mathrm{n}}
\end{gathered}
$$

Although the approximation greatly reduces the utility of this result, we feel that the general form of the resultant equation is still useful, and the approximate solution provides a check on the reasonableness of later results. Based on this derivation, we chose to use the model $\sigma(t)=\sigma_{0}-\sigma_{1} t^{n}$ in our efforts to model stress relaxation behavior.

The other method of obtaining a stress relaxation model from creep data is to begin by performing the viscoelastic conversion upon the data sets by the use of the convolution integral $\int E(t) D(t-T) d T=t$. We performed this 
Stress Relaxation Data and Converted Creep Data

Kapton of $55 \mathrm{C}$

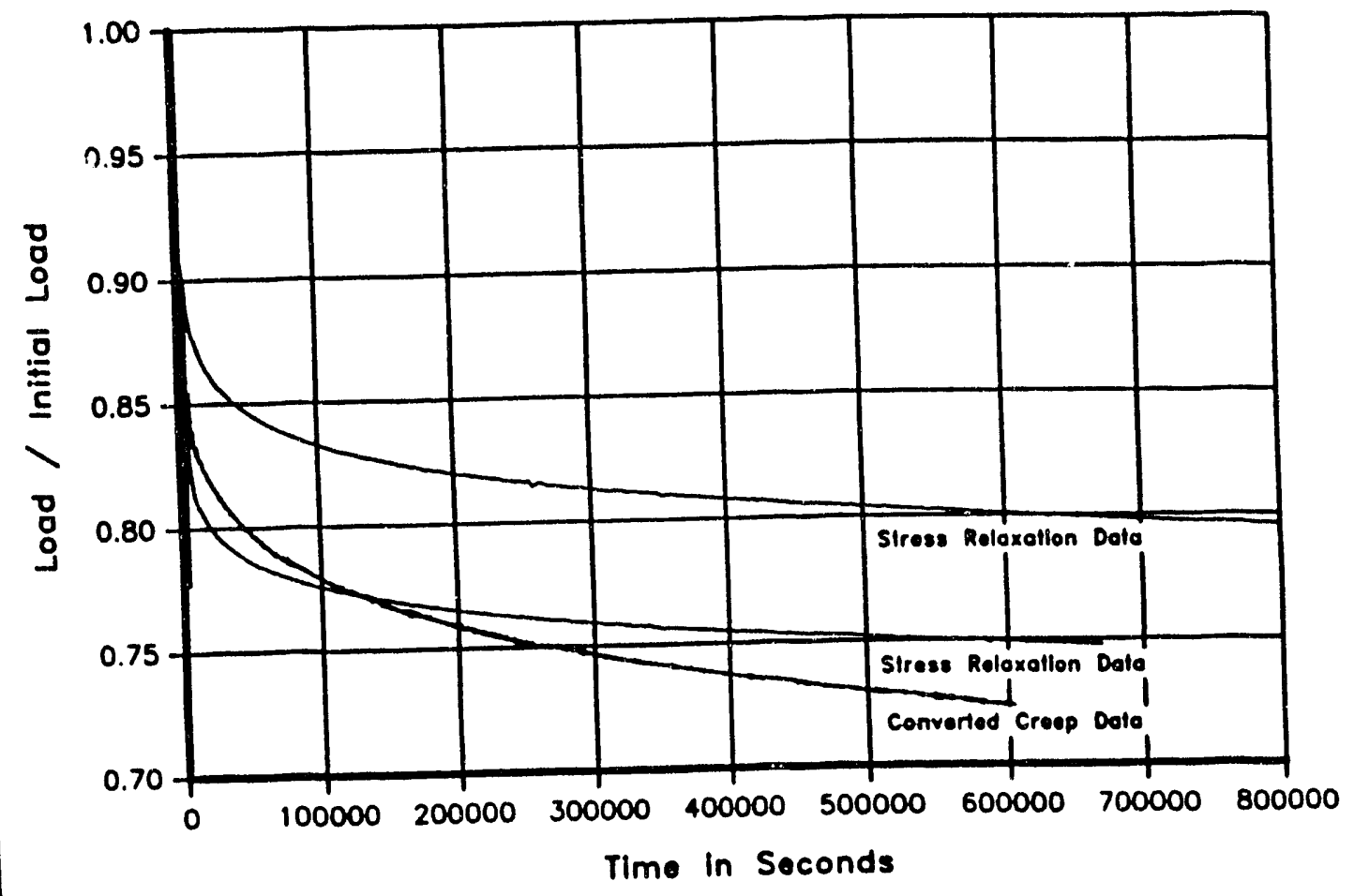

Figure 3: Comparison of Stress Relaxation data and Creep Equivalent.

data gather from stress relaxation experiments at the same temperature. The converted creep data exhibited a much steeper slope than the stress relaxation data. This suggests that while the creep data cannot be used as an accurate predictor of stress relaxation behavior, it should provide an upper bound for such behavior.

\section{REFERENCE}

1. Garafalo, "Fundamentals of Creep in Metals," Macmillan, New York, NY 1965.

2. F. J. Lockett, "Nonlinear Viscoelastic Solids," Academic Press, New York, NY 1972.

3. I. L. Hopkins and R. W. Hamming, "On Creep and Relaxation," Journal of Applied Physics, Vol. 28, p. 906, 1957.

4. John D. Ferry, "Viscoelastic Properties of Polymers," John Wiley \& Sons, New York, NY 1980. 
4. John D. Ferry, "Viscoelastic Properties of Polymers," John Wiley \& Sons, New York, NY 1980.

5. Lautenschlager, A., et al., "Temperature Dependence of the Viscoelastic Properties of SSC Coil Insulation (Kapton)", presented at 1992 IISSC Conference, New Orleans, LA, March 1992. 

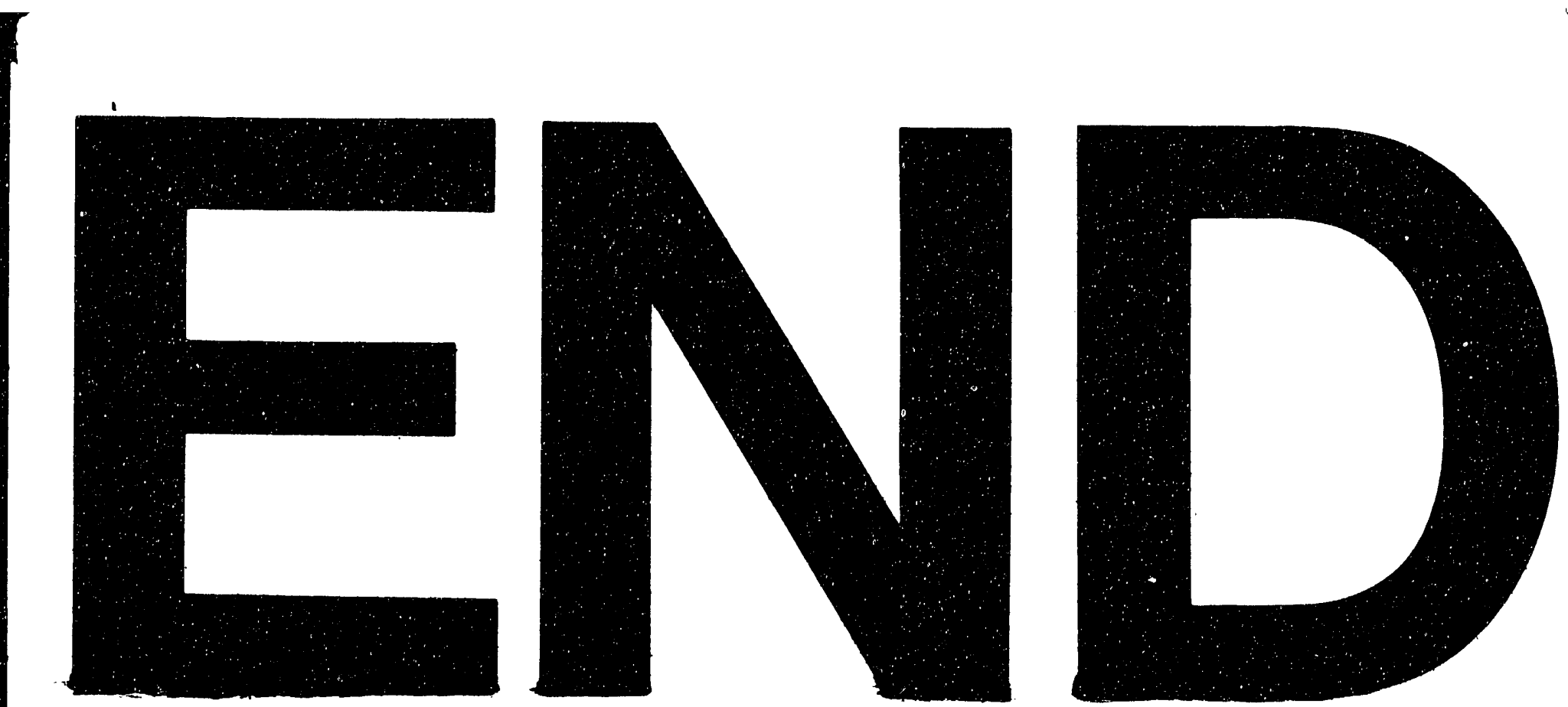

$F$

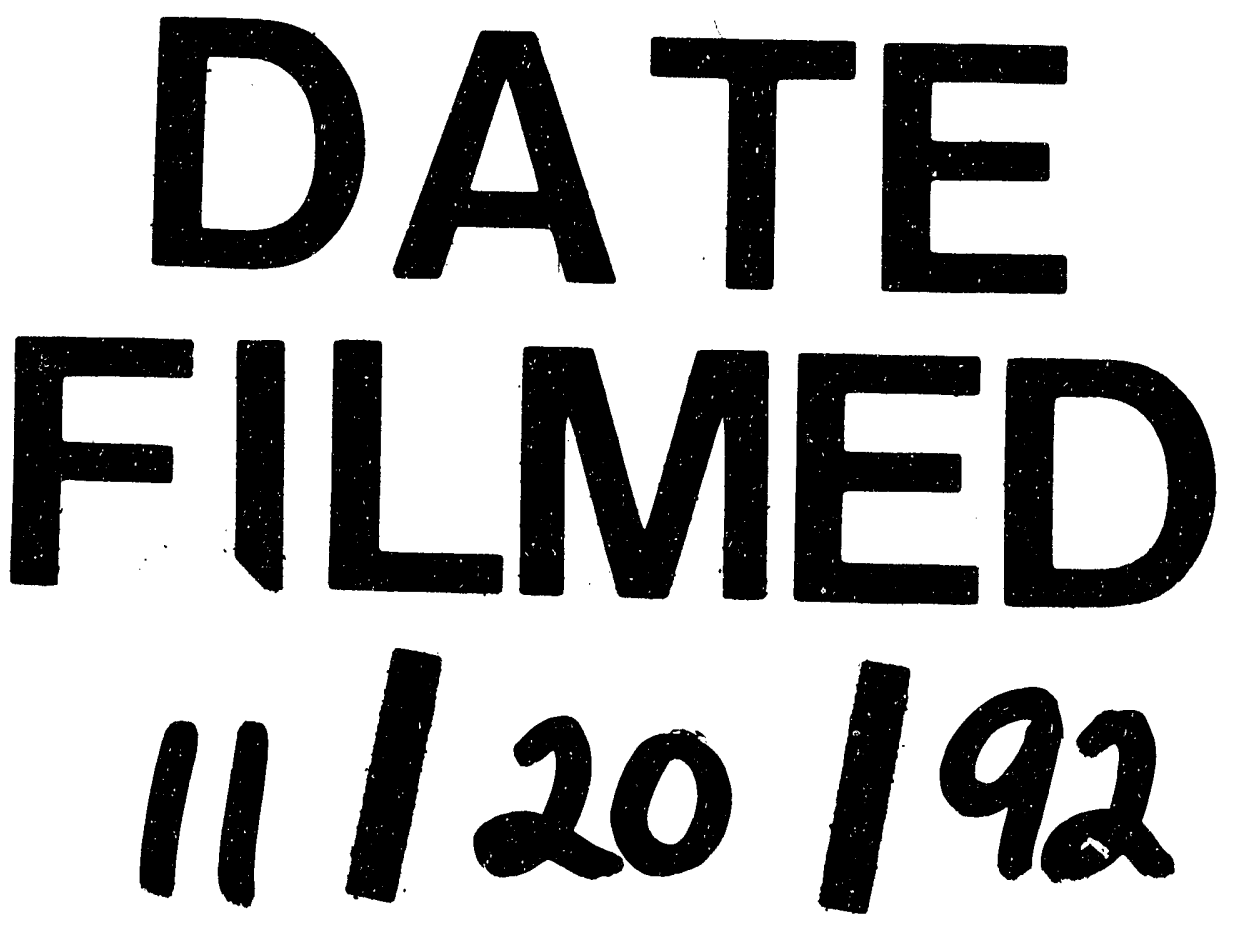




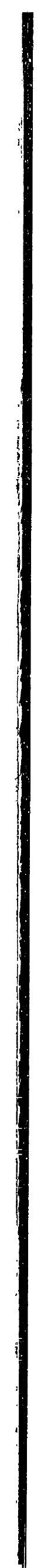

